


\section{About IJMA [last updated October, $\left.1^{\text {st }}, 2021\right]$}

$\checkmark$ International Journal of Medical Arts is the Official Journal of the Damietta Faculty of Medicine, AlAzhar University, Egypt

$\checkmark$ It is an International, Open Access, Double-blind, Peer-reviewed Journal

$\checkmark$ Published four times a year

$\checkmark$ The First Issue was published in July 2019

$\checkmark$ Published under the following license: Creative Commons Attribution-ShareAlike 4.0 International Public License (CC BY-SA 4.0). It had updated from the Creative Commons license [CC BY] in volume 2, Issue 4, October 2020 About IJMA

$\checkmark$ The Egyptian Knowledge Bank hosts the web site of IJMA

$\checkmark$ The Egyptian Knowledge Bank supports IJMA

$\checkmark$ IJMA follows the regulations of the International Committee of Medical Journal Editors

$\checkmark$ IJMA is indexed in the "Directory of Open Access Journals" [15 January 2021].

$\checkmark$ IJMA is indexed in J-Gate [29-6-2021]

$\checkmark$ IJMA is a member of the International Society of Managing and Technical Editors

$\checkmark$ Listed in "Index Copernicus", "Publons", "Academic resource index [ResearchBib]" "Electronics journal library", "Eurasian Scientific Journal Index", "WorldCat" Superstar Journal Database, and "Citefactor"

$\checkmark$ IJMA introduced to the search engine [BASE] through DOAJ

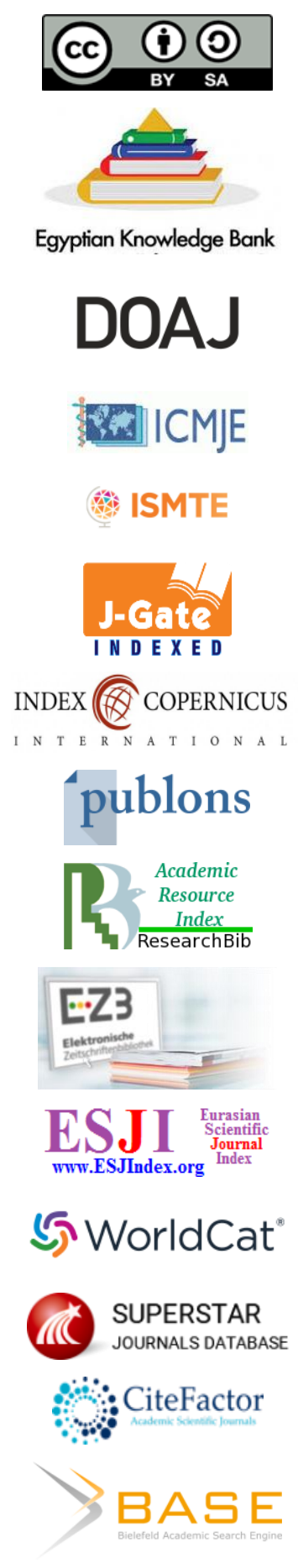




Main subject [Dermatology and Venereology]

Original Article

\title{
Evaluation of Efficacy and Safety Topical 5-Fluorouracil $0.5 \%$ in Treatment of Resistant Vitiligo [Alone or After Microneedling]; A pilot Study.
}

\author{
Sara Ahmed Galal [1], Moshiraly Mohammed Ali [1], Fatma Refaat Elzeiny Elzeiny [2]. \\ 1 Department of Dermatology and Venereology, Faculty of Medicine for Girls, Al-Azhar University, Egypt. \\ 2 Third Doctor in the Medical Organization [for Girls], Al-Azhar University, Cairo, Egypt.
}

Corresponding author: Sara Ahmed Galal

Email: drsaragalal@azhar.edu.eg

Submission date: August 04, 2021; Acceptance date: September 07, 2021

DOI: $10.21608 / I J M A .2021 .89242 .1347$

DOAJ

\section{ABSTRACT}

Background: Vitiligo is a chronic acquired disorder characterized by the depigmented macules. There are many treatment options, but the response is variable and unsatisfactory. Topical 5-fluorouracil used in many dermatological diseases but, this is the first study to date that use $0.5 \%$ concentration in vitiligo treatment.

The Aim of The Work: This study evaluates efficacy and safety topical 5 -fluorouracil in $0.5 \%$ concentration in treatment of resistant vitiligo either alone or after microneedling.

Subject and Methods: 30 patients with resistant vitiligo were enrolled into the study; we studied only 2 patches in every patient. The first patch treated with one session of microneedling followed by topical 5 -fluorouracil $0.5 \%$ once daily for 2 weeks. The second patch treated with topical 5 -fluorouracil $0.5 \%$ only for 2 weeks, then follow up of both patches for 3 months.

Results: Excellent response achieved in $46.7 \%$ of the patches treated with microneedling followed by topical 5 -fluorouracil $0.5 \%$, while only in $23.3 \%$ of the patches treated with topical 5 -fluorouracil $0.5 \%$ alone. There was no significant difference between both groups regarding response, despite this the degree of improvement was higher in combined treatment.

Conclusion: Topical 5 -fluorouracil $0.5 \%$ is an effective and safe in treatment of resistant vitiligo and combined therapy increases the efficacy.

Keywords: 5-fluorouracil 0.5\%; Microneedling; Pigmentation; Vitiligo.

This is an open-access article registered under the Creative Commons, ShareAlike 4.0 International license [CC BY-SA 4.0] [https://creativecommons.org/licenses/by-sa/4.0/legalcode.

Citation: Galal SA, Ali MM, Elzeiny FRE. Evaluation of Efficacy and Safety Topical 5-Fluorouracil 0.5\% in Treatment of Resistant Vitiligo [Alone or After Microneedling]; A pilot study. IJMA 2021; 3 [4] October-December: 1803-1810. [DOI: 10.21608/IJMA.2021.89242.1347].

* Main subject and any subcategories have been classified according to the research topic 


\section{INTRODUCTION}

Vitiligo is an acquired localized depigmentation. It results from the progressive loss of melanocytes. It appears as milky white macules sharply demarcated ${ }^{[1]}$. It affects $1 \%$ of the world's population of any age and sex ${ }^{[2]}$.

Vitiligo is a multifactorial disease; many hypotheses have been suggested for its etiology [3]. Melanocyte damage occur by different ways leading to vitiligo, ROS, autoantibodies, auto-reactive $T$ cells along with genetic background are implicated in melanocyte loss [4]. Many theories implicated in the etiology of vitiligo; autoimmune hypothesis is supported by the association of vitiligo with other autoimmune disorders such as thyroid disease [1].

Different therapy used to induce repigmentation of skin by stimulation of proliferation of remaining melanocytes around the hair follicles and melanocyte at the border of lesions, so the goal of the treatment is to stimulate melanocyte migration and stop their destruction [5]. However, patients are resistant to medical treatment, various surgical modalities can be used either alone or in conjunction with medical treatment in stable recalcitrant vitiligo [2]

5-fluorouracil is pyrimidine analog has antimetabolite activity ${ }^{[6]}$. Topical application of 5 -fluorouracil $5 \%$ as single therapy or combined with other modalities reported in the literature to helps repigmentation ${ }^{[7-9]}$.

In the present study, we are evaluating the effect of topical 5 -fluorouracil $0.5 \%$ in treatment of resistant vitiligo either alone or combined with microneedling. This is the first study to date that use topical 5- fluorouracil in concentration $0.5 \%$ not $5 \%$ to increase response rate and avoid the side effects in resistant vitiligo.

\section{PATIENTS AND METHODS}

This prospective, comparative clinical pilot trial included 30 patients with resistant vitiligo. The patients were recruited from outpatient clinic of Al-Zahraa University Hospital, Faculty of medicine for girls, Al-Azhar University during the period from January 2017 to January 2018. Informed written consent was obtained from all patients. The approval from the Research Ethics Committee of the faculty of medicine, Al-Azhar University was also obtained.

\section{Patients}

Patients with resistant and stable vitiligo; no increase in number or size of the present lesions for at least 6 months were included in the current study. The treated patients had at least 2 stable vitiliginous patches. Patients less than 18 years were excluded. Alopecia areata patients and patients on other treatments for vitiligo for at least one month before the study were also excluded. Pregnant and lactating women, patients with history of koebner phenomenon, impaired wound healing, bleeding tendency, impaired wound healing, history of hepatitis B or C, HIV and other uncontrolled chronic systemic diseases were also excluded from the study.

\section{Treatment protocol:}

Explanation of the procedure to all patient included in this study with all possible cosmetic procedure results and occurrence of possible complications, whether transient or persistent and consent was obtained. Photograph was taken for the patients using camera Olympus c-420 digital SLR camera 13MP before starting the treatment and during the follow up period.

\section{Each patient has two vitiliginous patches:}

The first patch; was treated by topical 5-fluorouracil $0.5 \%$ which applied once daily under occlusion.

The other patch was subjected to microneedling with dermapen followed by application of 5 -fluorouracil $0.5 \%$ once daily under occlusion. A topical anesthetic cream [Pridocaine ${ }^{\circledR}$ a combination of two local anesthetics lidocaine and prilocaine] was applied under occlusion on the patch 30 minutes before treatment to reduce the pain, then it was washed off and sterilized with gauzes soaked in $70 \%$ isopropyl alcohol. Microneedling was performed to one patch with dermapen [4000 turn per minute of Dr. Pen Ultima-A1] at the highest speed with penetration depth at 2 $\mathrm{mm}$ in uniform horizontal and vertical directions until pinpoint bleeding was observed. Topical 5-fluorouracil cream $0.5 \%$ then was applied under occlusive dressing once daily for 2 weeks.

\section{Assessments:}

Photographs were obtained at baseline, before starting treatment session, and 3 months after the final treatment. 2 blinded dermatologists were asked for objective clinical assessment at the baseline and 3 months after the last session for the degree of repigmentation.

The assessment was done according to Investigator's global assessment [IGA] ${ }^{[10]}$, as the following; No change, poor improvement $=$ [less than $25 \%$ ], good improvement $=$ $[26 \%-50 \%]$, very good improvement $=[51 \%-75 \%]$ and excellent improvement $=[76 \%-100 \%]$. 
Patient's satisfaction; Patient's satisfaction was performed 3 months after the last treatment session using for the ease and the degree of improvement 3 months after treatment as follows; 0 : no satisfaction, 1: poorly satisfied, 2: moderate satisfaction, 3: fully satisfied. Patients were evaluated clinically for the improvement and the side effects after the treatment session, one month and three months after the last sessions. Side effects include, erythema, edema, hyperpigmentation, hypopigmentation, koebner phenomenon and scarring.

Statistical analysis: Data were collected, revised, coded and entered to the Statistical Package for Social Science [IBM SPSS] version 23. The quantitative data were presented as mean, standard deviations and ranges when their distribution found parametric, the comparison between groups with qualitative data were done by using Chi-square test and Fisher exact test instead of the Chi-square only when the expected count in any cell found less than 5 . The confidence interval was set to $95 \%$ and the margin of error accepted was set to $5 \%$. So, the $P<0.05$ was considered significant.

\section{RESULTS}

This study comprised 30 patients with resistant vitiligo, 12 of the patients [40\%] were males and 18 [60\%] were females. Their ages ranged from $18-60$ years [mean \pm SD: $37.93 \pm 12.97$ years]. Concerning duration of vitiligo in patients it was ranged from 3 years to 20 years with a median value [IQR] 8.5. Ten out of thirty patients [33.3\%] had positive family history of vitiligo and 20 patients [66.7\%] had negative family history.

The clinical type: 17 patients [56.7\%] were nonsegmental vitiligo, while 13 [43.3\%] were segmental vitiligo. Most of patients 25 were stable for one year [83.3\%] and leukotrichia was reported in 7 patients [23.3\%].

Regarding Fitzpatrick skin types of the studied patients one patient [3.3\%] was skin Type II, 14 patients [46.7\%] with type III and 15 patients [50.0\%] were skin type IV. Sixty resistant vitiligo patches were studied in this study distributed over the face [ $n=4]$, trunk [ $n=28]$, extremities [ $n$ $=36]$ and acrofacial $[n=6]$. The overall qualitative response was better in the patches treated with 5 -fluorouracil $0.5 \%$ after dermapen than the patches treated with 5 -fluorouracil $0.5 \%$ only. In the patches treated with topical 5 -fluorouracil $0.5 \%$ after needling; 14 patches [46.7\%] showed excellent response, 3 [10\%] showed very good response, 3 [10\%] showed good response and 10 patches [33.3\%] showed poor response. On the other hand, in the patches treated with topical 5- fluorouracil $0.5 \%$ only; 7 patches [23.3\%] showed excellent response, 4 [13.3\%] showed very good response, 6 [20\%] showed good response, 13 patches [43.3\%] showed poor response. There is no statistically significant difference between both groups [Table 1].

[Figures 1-3] show photographic examples of before vs. after treatment photos of the participants. There was no statistically significant difference as regard patients' satisfaction of in both groups. The pattern of pigmentation was the same in both groups; in which 21 patients [70\%] showed diffuse pattern of repigmented, while 6 patients $[20 \%]$ showed follicular pattern and only 3 patients $[10 \%]$ did not develop any pigmentation at all. There is no statistically significant difference between pattern of repigmentation and degree of improvement. There was no statistically significant correlation between degree of improvement in both groups as regard their age, sex, pattern of the disease and positive family history with P-value $>0.05$. There were also no statistically significant correlations between degree of improvement and site of the lesion, association with leukotrichia, stability and skin type. There was statistically significant negative correlation between the degree of improvement in patches treated with 5 -fluorouracil $0.5 \%$ after dermapen and the duration of the disease $p$-value [0.028] [Figures 4]. While there was no statistically significant correlation between them in patches treated with 5 -fluorouracil $0.5 \%$ only.

Regarding the Side effects, most patients 27 patient [90\%] did not develop any complication, only 2 patients [6.7\%] showed hyperpigmentation and Koebner phenomenon was recognized in one patient [3.3\%] [Figures $4,6]$. There was no clinical manifestation of systemic toxicity reported. There was no statistically significant correlation between degree of improvement and susceptibility to complications in both patches.

Table [1]: comparison between repigmentation in both groups.

\begin{tabular}{|c|c|c|c|c|c|c|}
\hline \multirow[t]{2}{*}{ Repigmentation } & \multicolumn{2}{|c|}{$\begin{array}{c}\text { Group A } \\
{[5-\mathrm{FU} 0.5 \%+\text { microneedling] }}\end{array}$} & \multicolumn{2}{|c|}{$\begin{array}{l}\text { Group B } \\
{[5-F U ~ 0.5 \%]}\end{array}$} & \multirow[t]{2}{*}{ Test value } & \multirow[t]{2}{*}{ P-value } \\
\hline & No. & $\%$ & No. & $\%$ & & \\
\hline Poor & 10 & $33.3 \%$ & 13 & $43.3 \%$ & 0.635 & 0.426 \\
\hline Good & 3 & $10.0 \%$ & 6 & $20.0 \%$ & 1.176 & 0.278 \\
\hline Very Good & 3 & $10.0 \%$ & 4 & $13.3 \%$ & 0.162 & 0.687 \\
\hline Excellent & 14 & $46.7 \%$ & 7 & $23.3 \%$ & 3.590 & 0.058 \\
\hline
\end{tabular}


No. = Number, 5-FU= 5-fluorouracil

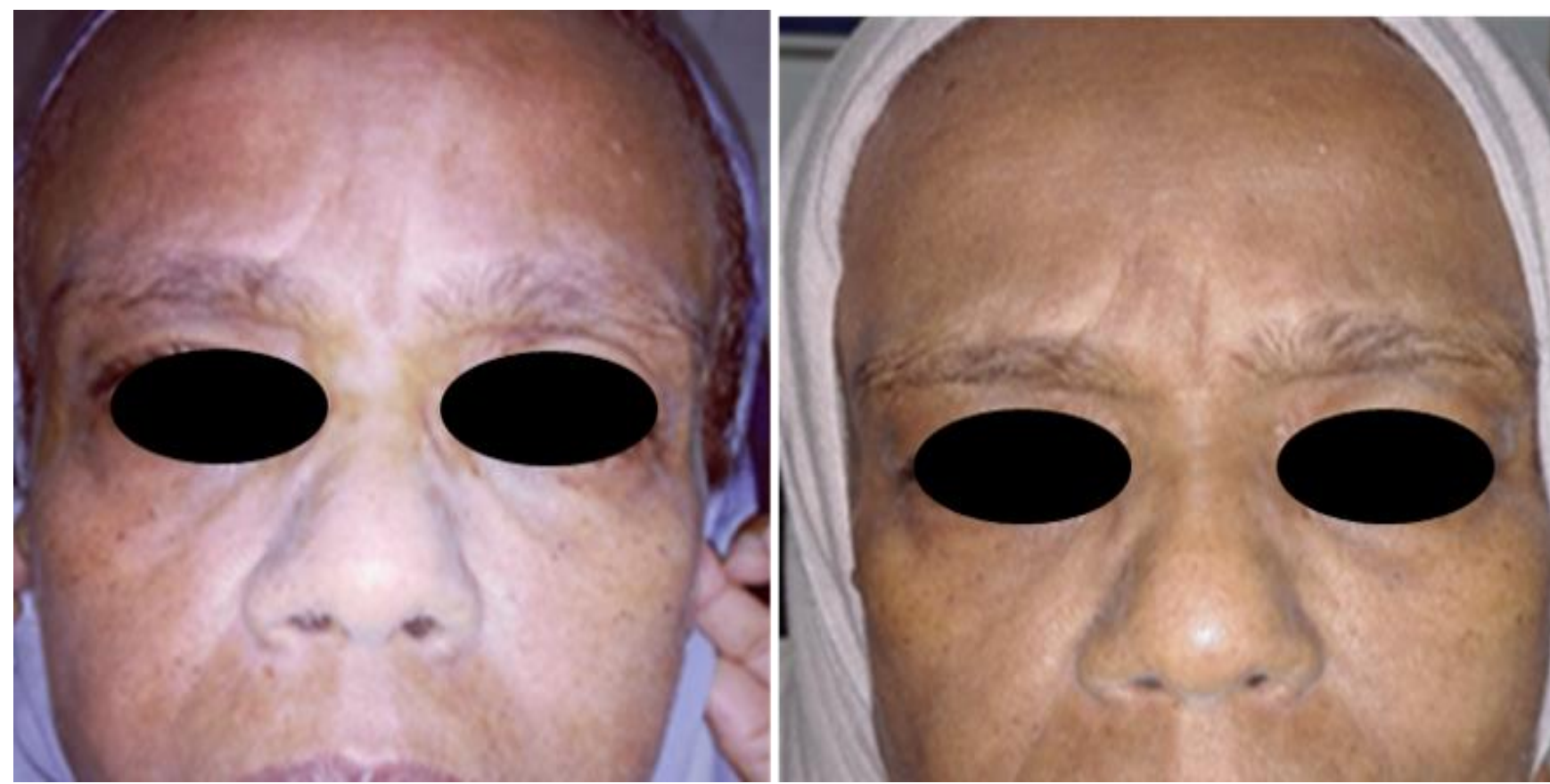

Figure [1]: Facial vitiligo, $[A]$ before treatment. $[B]$ Both sides show excellent response after treatment [right side treated with dermapen followed by fluorouracil $0.5 \%$ and, the left side treated with topical 5 - fluorouracil $0.5 \%$ only

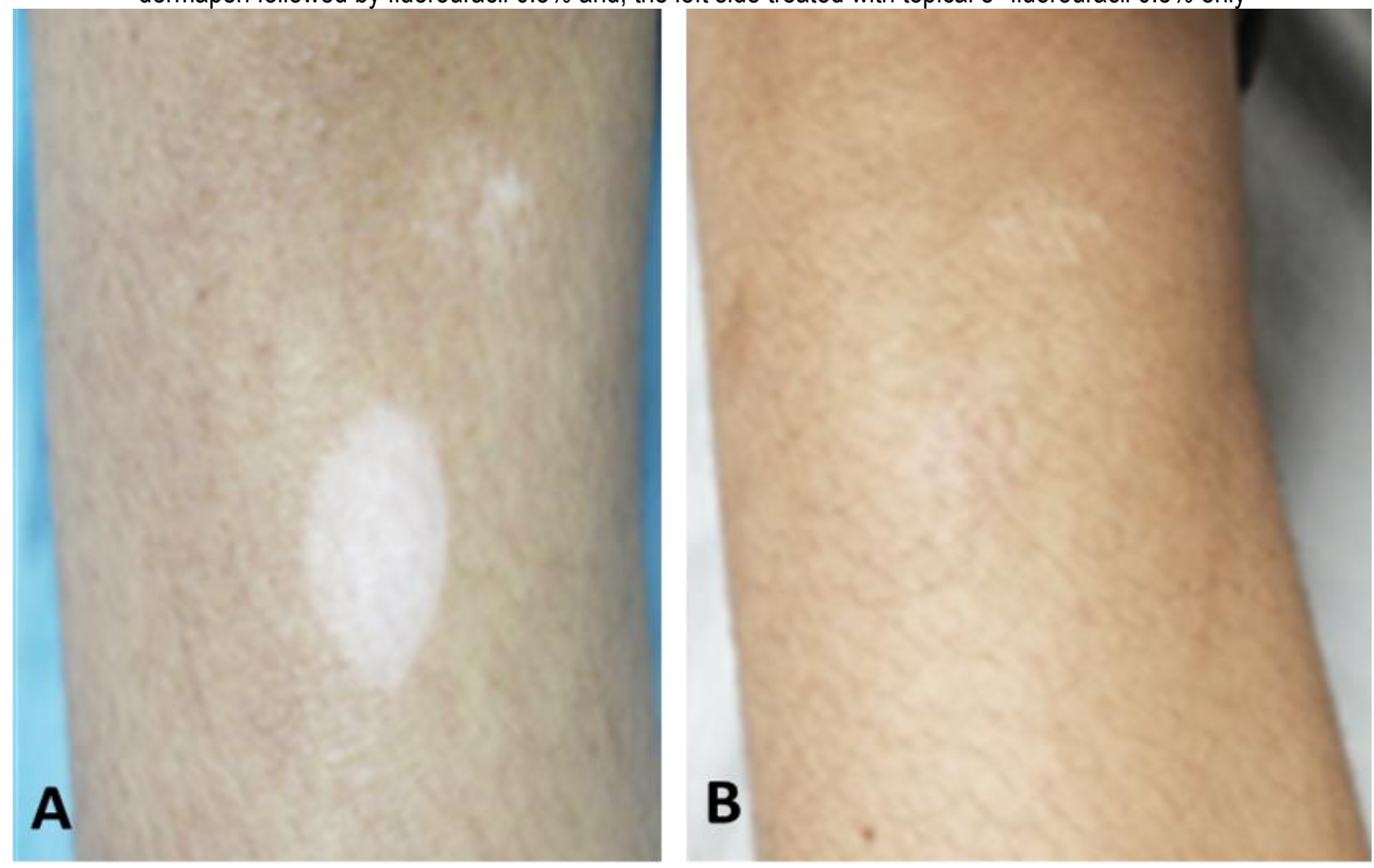

Figure [2]: Vitiligo patch on the shaft of the tibia [A] before treatment $[B]$ after treatment with dermapen followed by topical 5 - fluorouracil $0.5 \%$ the case shows excellent repigmentation 


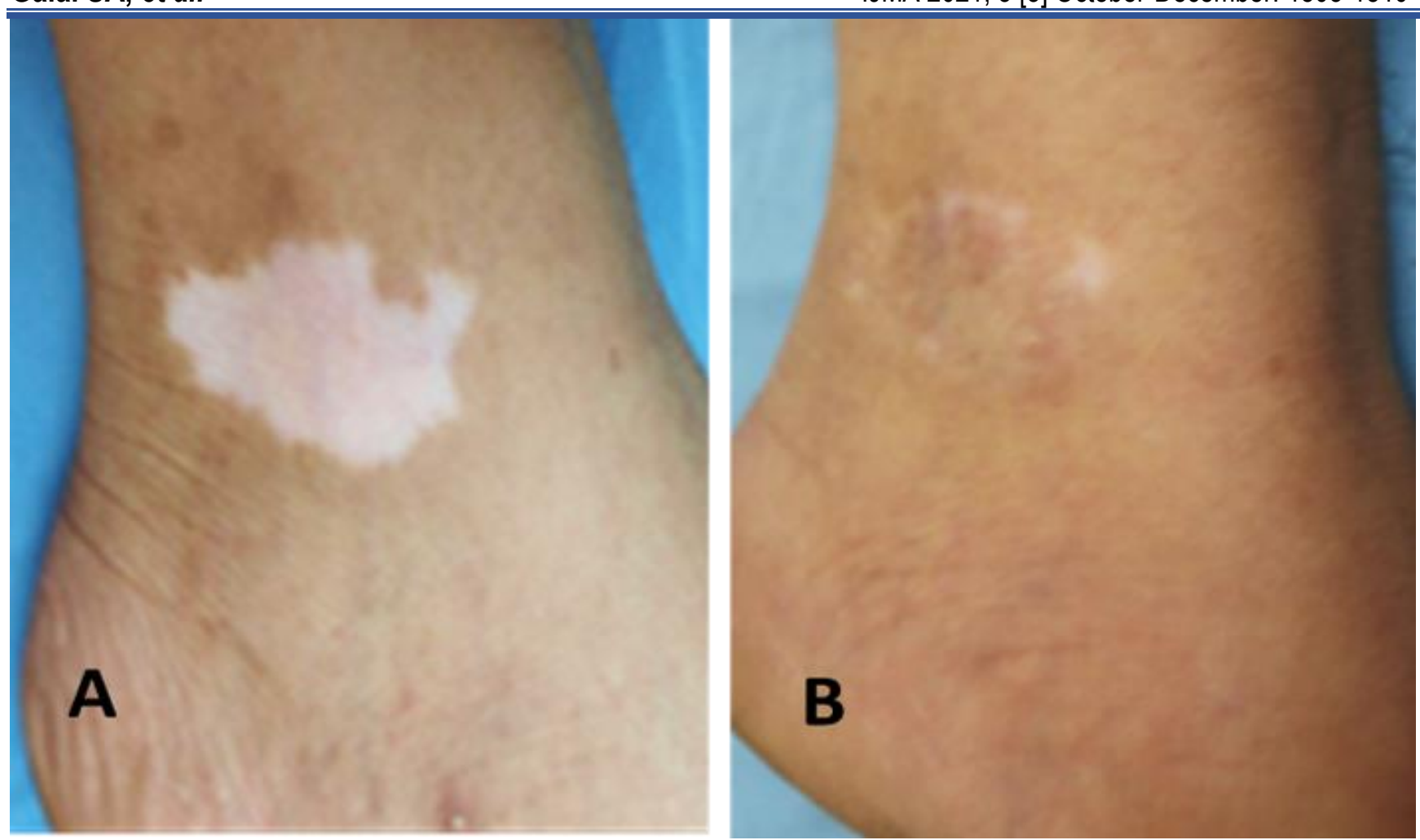

Figure [3]: Vitiligo patch over medial malleolus of right leg $[\mathrm{A}]$ before treatment $[\mathrm{B}]$ after treatment with topical fluorouracil $0.5 \%$ shows excellent response.

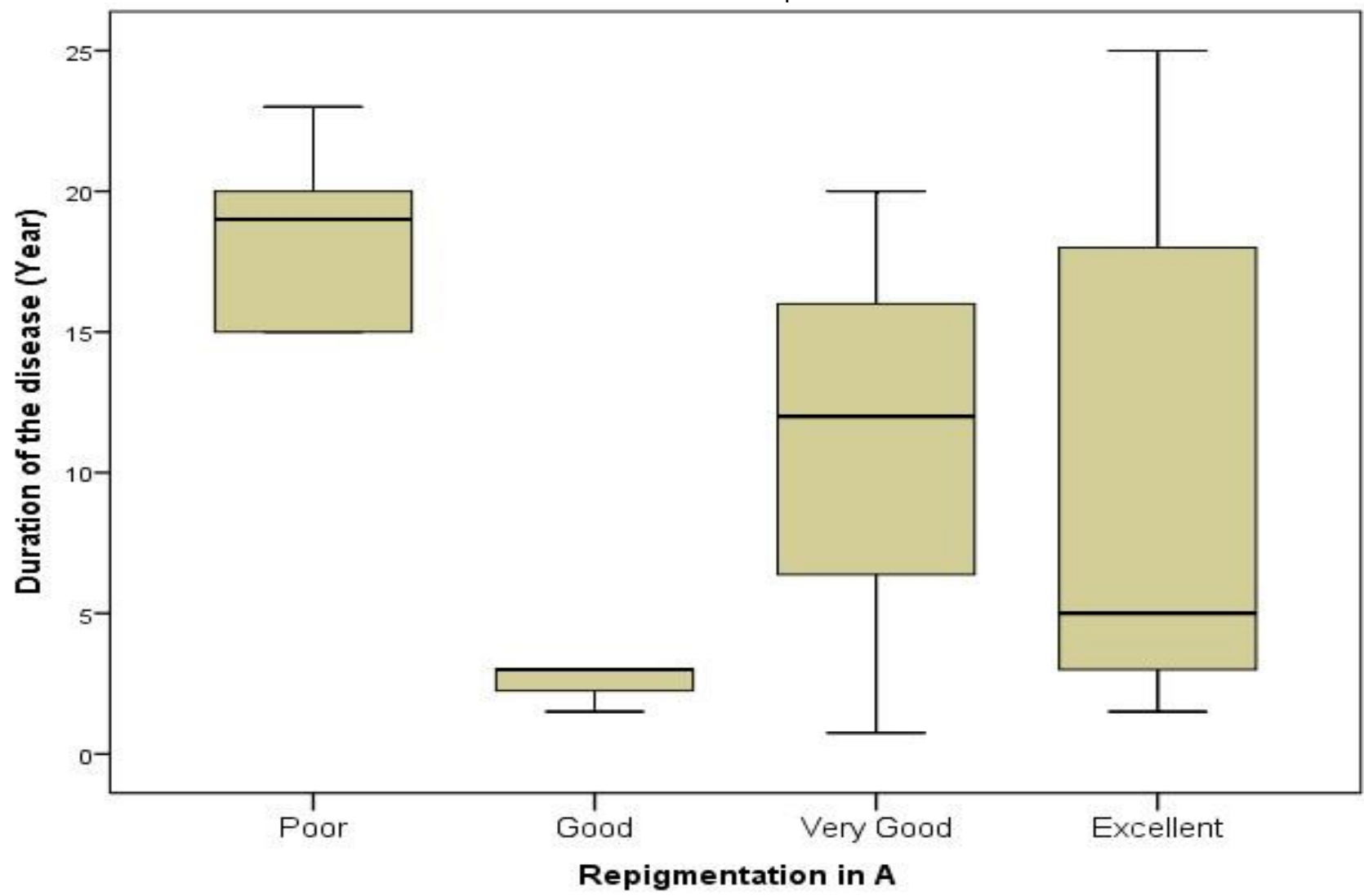

Figure [4]: Statistically significant negative correlation between degree of re-pigmentation and the duration of the disease in in the patients treated 5 -fluorouracil $0.5 \%$ after dermapen 


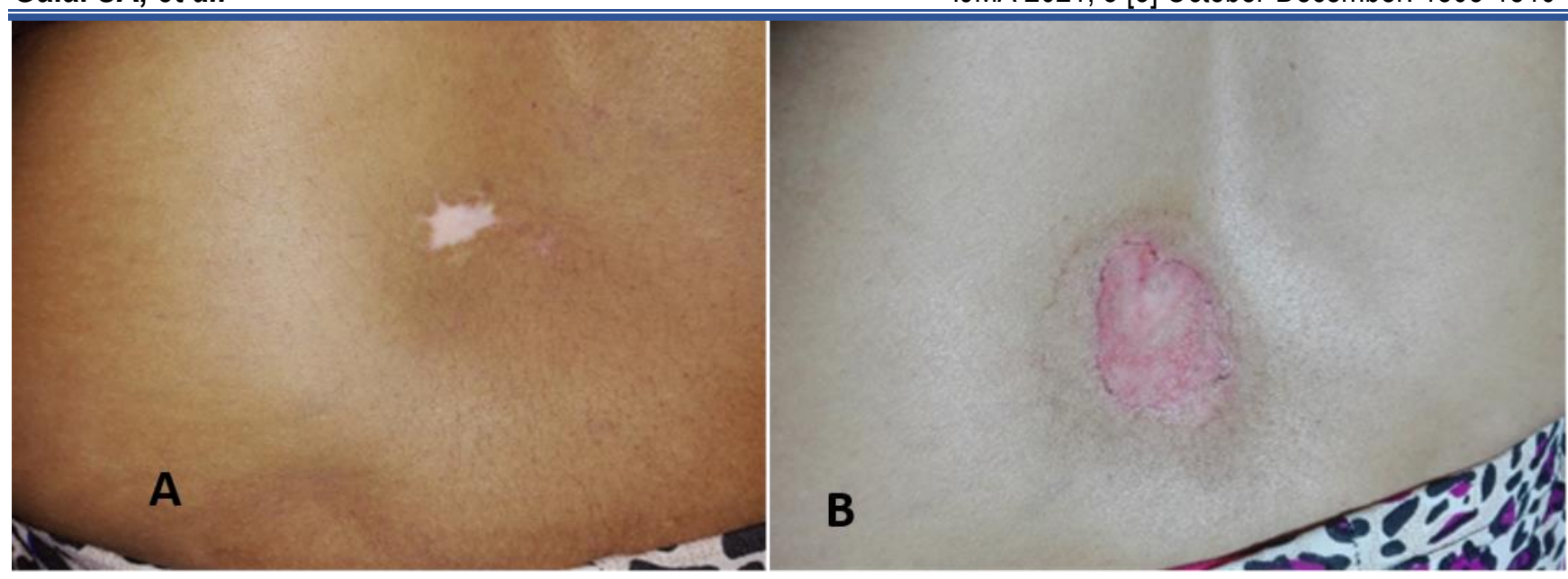

Figure [5]: localized vitiligo on the lower part of the back the vitiliginous patch treated with dermapen +5 - fluorouracil $0.5 \%$ shows erythema and widening of the patch [kobenerization].
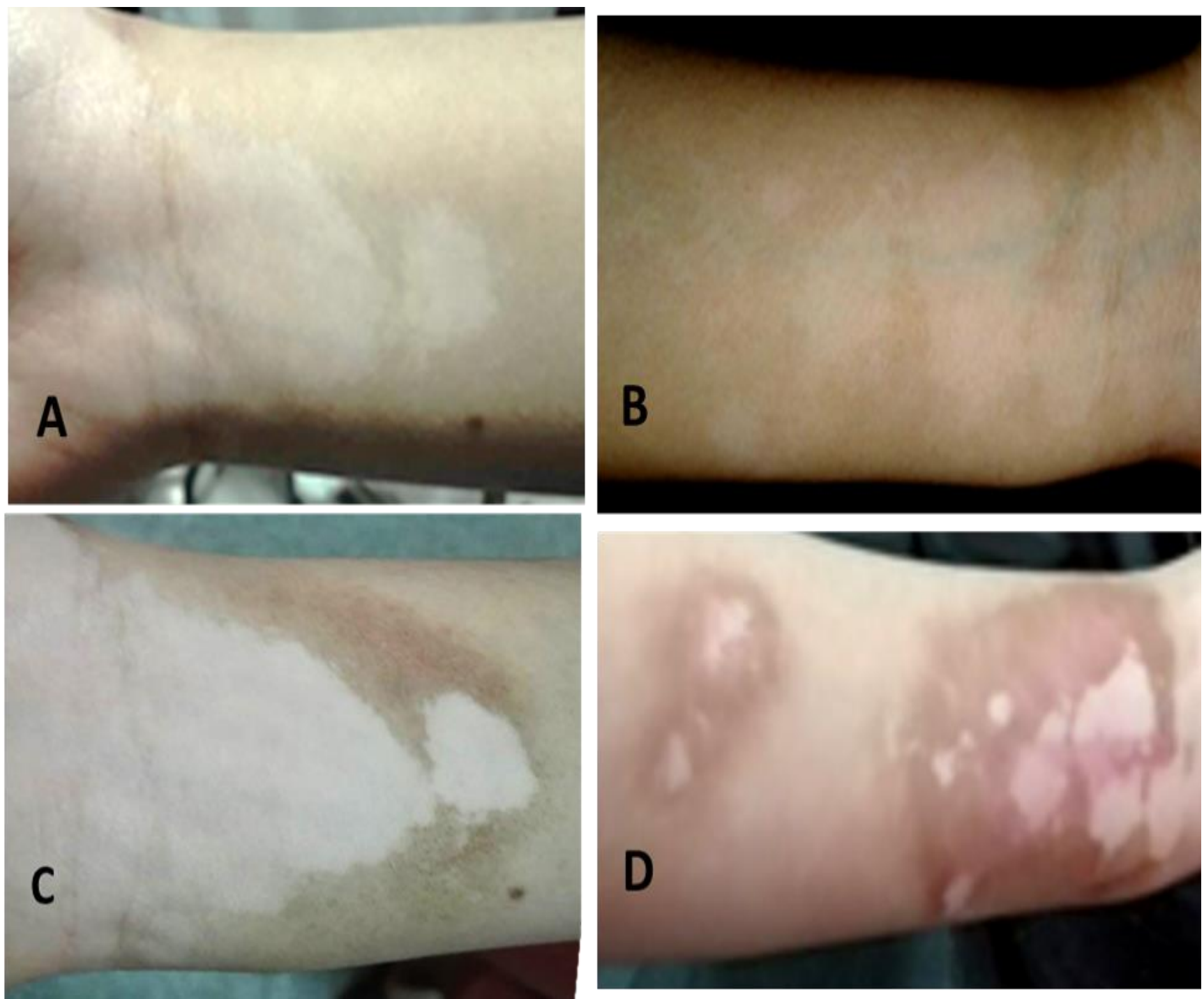

Figure [6]: female patient with vitiligo: a vitiliginous patches in the right arm and left arm $[A, B]$. The patient developed post inflammatory hyperpigmentation in both forearms [C, D]. [The left arm after treatment with dermapen followed by 5 - fluorouracil $0.5 \%[C]$ and the right arm after treatment with 5 - fluorouracil only [D]. 


\section{DISCUSSION}

Vitiligo is challenging disease with variable results [11], as no single therapy can give satisfactory results with all patients [12]. Trials demonstrated that combined treatment could increase the overall response with decrease both the duration of treatment and the possible side effects [11].

Topical $5 \%$ fluorouracil is not very common for the treatment of vitiligo [13]. Tsuji and Hamada ${ }^{[7]}$ was the first who introduced combination of therapeutic wounding and topical 5-fluorouracil as treatment for vitiligo in 1983.

This study was conducted on 30 patients with resistant vitiligo, two vitiliginous patches were treated only in each patient; one of them was treated with topical 5- fluorouracil $0.5 \%$ combined with microneedling and the other patch was treated with topical 5 - fluorouracil $0.5 \%$ only.

In this study there was very good to excellent response in $56.7 \%$ of the patients treated with combined dermapen with 5 -fluorouracil $0.5 \%$, while in $36.6 \%$ patients in the treated with topical 5 - fluorouracil $0.5 \%$ only. There was no statistically significant difference between both groups; despite this the degree of improvement was higher in group treated with 5 - fluorouracil $0.5 \%$ after needling. Combination of dermapen with 5 -fluorouracil $0.5 \%$, is a promising treatment resistant vitiligo.

Although the idea of combination therapy is not a new approach for treatment of vitiligo, this is the first study to date that used topical 5- fluorouracil in concentration $0.5 \%$ not $5 \%$. Our result is almost the same to the studies that used topical 5 - fluorouracil in concentration $5 \%$ as Shashikiran et al. ${ }^{[13]}$ study in which topical $5 \%$ fluorouracil needling showed good to excellent response in $75 \%$ of the patches and in Yones et al. [12] study almost $45 \%$ of patients achieved excellent repigmentation after mechanical dermabrasion. Also, Tsuji and Hamada [7] study $64 \%$ showed complete or almost complete repigmentation in all spots treated with 5\% fluorouracil cream daily under occlusive dressings, following an epidermal abrasion on the first day.

While Vedamurthy et al. ${ }^{[2]}$ in his trial on three patients of resistant vitiligo, all patients showed excellent repigmentation, but the low number of cases and the different protocols of treatment these items make comparison of results is not reliable. There patients were subjected to needling with topical 5- fluorouracil 5\% application every two weeks for three months, along with their ongoing therapy. Interestingly Mohamed et al. ${ }^{[14]}$ study achieved good to excellent re-pigmentation in $55.9 \%$ of the acral vitiligo lesions when topical 5 -fluorouracil $5 \%$ used in combination carbon dioxide $\left[\mathrm{CO}_{2}\right]$ laser, while when used alone it achieved good to excellent re-pigmentation in $5.7 \%$ only. Also, Asker et al. [10] trial results were not promising either by 5 -Fluorouracil alone or after manual abrading of the lesion with sandpaper. This may be interpreted by the different concentration used of 5-Fluorouracil. The more resistance acral area also may play roles due to lower density of pilosebaceous unite, melanocyte and melanocyte stem cell reservoirs ${ }^{[10,15]}$.

This is the first study to date that use topical 5fluorouracil in concentration $0.5 \%$ not $5 \%$ in vitiligo. This lower concentration not only avoids the side effects of higher concentrations of 5- fluorouracil in vitiligo but also may be a method of improving response rate and hasten the duration of therapy. This explained by levy et al. ${ }^{[16]}$ study which interestingly, reported that fluorouracil were retained in a higher percentages in the skin after topical application of 0.5 $\%$ cream when compared with $5 \%$ cream which may indicates that the delivery of the $0.5 \%$ cream may be focused to the affected area. Therefore, this suggest that 0.5 $\%$ fluorouracil cream concentration may be more effective, safer and more tolerable.

Possible side effects of 5-FU which may be reported include pain, edema, blistering, erythema, ulceration, necrosis, scarring and hyperpigmentation or hypopigmentation [17]. Also itching was reported due to irritation of topical 5-FU [11, 10].

In this study most of our patients $90 \%$ did not develop any complication in both groups, but $6.7 \%$ showed hyperpigmentation and only one patient $3.3 \%$ showed koebnerization. Sethi et al. [9] study reported hyperpigmentation in $90 \%$ of patches treated with dermabrasion combined with 5-fluorouracil. Also, Asker et al. [10] study reported transient hyperpigmentation all [100\%]. These studies had higher rate of hyperpigmentation due to higher concentration of 5 - fluorouracil as they use $5 \%$ while in our study, we use $0.5 \%$.

Hrushesky [18] stated that hyperpigmentation is a common side-effect of 5 -fluorouracil, observed during the treatment of skin tumors and psoriasis, so we suggest that hyperpigmentation is related to the 5-fluorouracil and not complication of microneedling as it in reported both patches.

In the patient who developed koebnerization after both type of treatment within the 3 months of follow up, with further follow up in outpatient's clinic we noticed that complete repigmentation occurred in fourth and fifth month. Scarring was not reported in our study because of superficial needling process, and application of 5- fluorouracil cream once daily in very low concentration $0.5 \%$. 
Regarding safety topical 5-fluorouracil is absorbed to the systemic circulation in a very low amount. The amount of 5fluorouracil excreted in the patients' urine after using 5fluorouracil cream $0.5 \%$ was one fortieth that when the patients treated with the $5 \%$ cream [16]. Indicating that fluorouracil $0.5 \%$ cream may be a safer and as effective at $5 \%$.

According to this study, we conclude that topical 5fluorouracil in $0.5 \%$ concentration is as effective as $5 \%$ in treatment of resistant vitiligo and having advantage of being safer. Combination treatment with dermapen increases the effectiveness of topical 5- fluorouracil than when it used alone.

Limitations of this study were the lack of control lesions, wood light examination and lack of follow-up. Further intraindividual studies with different concentrations, longer follow-up periods, and immunohistochemical assessment are required to reach the optimal concentration.

\section{REFERENCES}

1- Santosh SK, Mohan SL, Gupta AK, Mohammad A, Kumar N. Treatment of Vitiligo with 5-Fluorouracil after Microneedling of the Lesion. Int J Sci Stud 2018; 5 [11]: 125-127. DOI: $10.17354 /$ ijss/2018/56

2- Vedamurthy M, Moorthy A, Samuel S. Successful treatment of vitiligo by needling with topical 5 fluorouracil. Pigment Disord 2016; 3:242. DOI: 10.4172/2376-0427.1000242

3- Begum R, Marfatia YS, Laddha NC, Dwivedi M, Mansuri MS, Singh M. Vitiligo: a complex disease and a complex approach. Mol Cytogenet. 2014 Jan 21; 7 [Suppl 1 Proceedings of the International Conference on Human]: 157. DOI: 10.1186/1755-8166-7-S1-I57.

4- Mansuri MS, Singh M, Jadeja SD, Gani AR, Patel R, Dwivedi M, Laddha NC, Begum R. Could ER Stress Be A Major Link Between Oxidative Stress And Autoimmunity In Vitiligo? Pigmentary Disorders 2014 1: 123. DOI:10.4172/ 2376-0427.1000123

5- Schallreuter KU, Krüger C, Würfel BA, Panske A, Wood JM. From basic research to the bedside: efficacy of topical treatment with pseudocatalase PC-KUS in 71 children with vitiligo. Int J Dermatol. 2008 Jul; 47 [7]:743-53. DOI: 10.1111/j.1365-4632.2008.03660.x.

6- Longley DB, Harkin DP, Johnston PG. 5-fluorouracil: mechanisms of action and clinical strategies. Nat Rev Cancer. 2003; 3[5]:330-8. DOI: 10.1038/nrc1074.

7- Abdelwahab M, Salah M, Samy N, Rabie A, Farrag A. Effect of Topical 5-Fluorouracil Alone versus Its Combination with Erbium: YAG [2940 nm] Laser in Treatment of Vitiligo. Clin Cosmet Investig Dermatol. 2020 Jan 23; 13:77-85. DOI: 10.2147/ CCID.S225881.
8- Gauthier Y, Anbar T, Lepreux S, Cario-André M, Benzekri L. Possible mechanisms by which topical 5-Fluorouracil and dermabrasion could induce pigment spread in vitiligo skin: an experimental study. ISRN Dermatol. 2013 Apr 9; 2013:852497. DOI: 10.1155/2013/852497.

9- Sethi S, Mahajan BB, Gupta RR, Ohri A. Comparative evaluation of the therapeutic efficacy of dermabrasion, dermabrasion combined with topical 5\% 5-fluorouracil cream, and dermabrasion combined with topical placentrex gel in localized stable vitiligo. Int J Dermatol 2007; 46:8759. DOI: 10.1111/j.1365-4632.2007.03226.x

10- Asker E, Mohammed GF, Gomaa AHA, Eyada MMK. Sandpaper and Topical 5-Fluorouracil: A Different Therapeutic Modality for Acral Vitiligo. ARC Journal of Dermatology 2019; [4] 1: 1-6. DOI: 10.20431/24560022.0401001

11- Ibrahim Z, Elgarhy L, Mina M. Comparison between the efficacy of microneedling combined with 5-fluorouracil vs microneedling with tacrolimus in the treatment of vitiligo. J Cosmet Dermatol. 2018; 00:1-8. DOI: 10.1111/jocd.12440

12- Yones AH, Mohamed EM, Mosbeh A, Tawfi KM. Efficacy of dermabrasion plus topical 5-fluorouracil in stable vitiligo. The Gulf Journal of Dermatology and Venereology 2013; 20[2]: 31-4.

13- Shashikiran AR, Gandhi S, Murugesh SB, Kusagur M; Sugareddy. Efficacy of topical $5 \%$ fluorouracil needling in vitiligo. Indian J Dermatol Venereol Leprol. 2018; 84 [2]: 203-205. DOI: 10.4103/ijdvl.IJDVL_386_16.

14- Mohamed HA, Mohammed GF, Gomaa AH, Eyada MM. Carbon dioxide laser plus topical 5-fluoro-uracil: a new combination therapeutic modality for acral vitiligo. J Cosmet Laser Ther. 2015; 17[4]: 216-23. DOI: 10.3109/14764172. 2014.1003241.

15- Mutalik S. Surgical management of acral vitiligo. In Somesh G, Mats O, Amrinder K, Jean-Paul O, eds. Surgical Management of Vitiligo, Blackwell publishing Ltd, Oxford, UK; 2007, 225-228.

16- Levy s, Furst K, Chern W. A novel $0.5 \%$ fluorouracil cream is minimally absorbed into the systemic circulation yet is as effective as $5 \%$ fluorouracil cream. Cutis 2002; 70[2]:14-21. PMID: 12353676

17- Kannambal K, Kaviarasan PK, Prasad PVS, Poorana B, Balajiganesh J. Efficacy of Intralesional 5- Fluorouracil in Recalcitrant Warts. Journal of medical science and clinical research 2019; 7:556-560. DOI: 10.18535/jmscr/v7i6.94.

18- Hrushesky WJ. Serpentine supravenous fluorouracil hyperpigmentation. J Am Med Assoc 1976; 236: 138. DOI: 10.1001/jama.1976. 03270020012002 


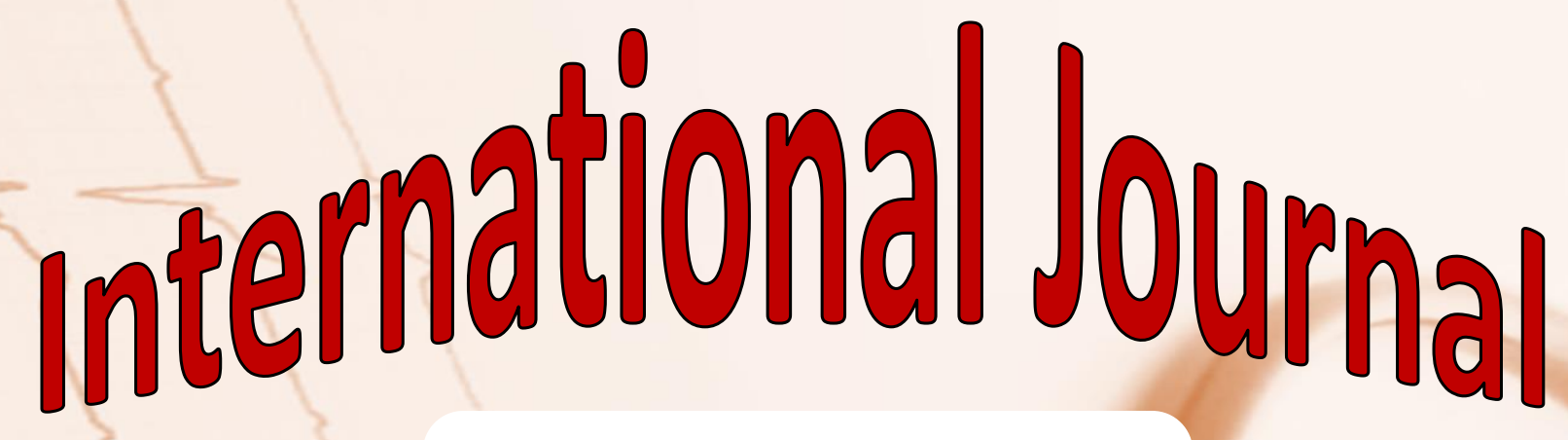

https://ijma.journals.ekb.eg/ Print ISSN: 2636-4174 Online ISSN: 2682-3780

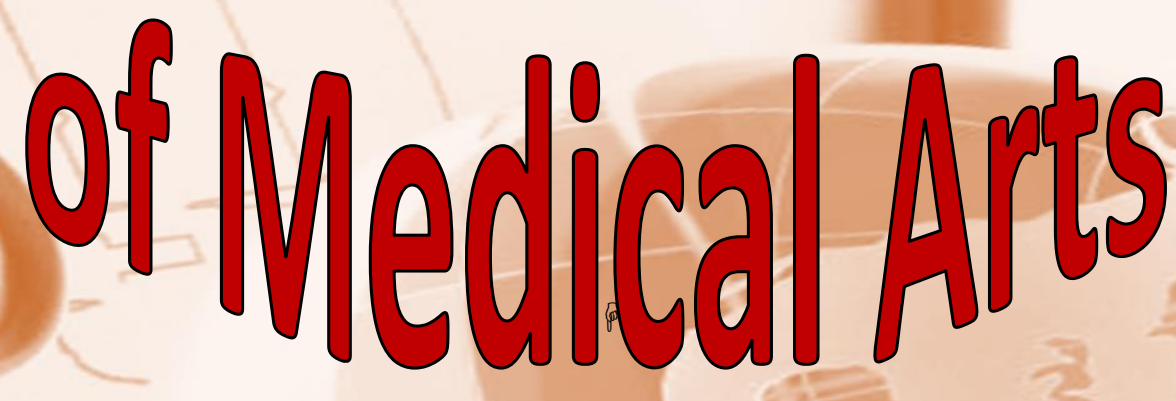

